\title{
The Virtual Lab Approach to Pharmacokinetics: Design Principles and Concepts
}

\author{
Wilhelm Huisinga*, Regina Telgmann, and Michael Wulkow
}

Dr. W. Huisinga*, DFG Research Center MATHEON \& Freie Universität Berlin, Fachbereich Mathematik und Informatik, Arnimallee 2-6, D-14195 Berlin, Phone: +49-30838 75119, Fax: +49-30-838 75412, e-mail: huisinga@ mi.fu-berlin.de, URL: http://compphysiol.mi.fu-berlin.de.

Dipl.-math. R. Telgmann, Dr. M. Wulkow, CiT GmbH, Oldenburger Str. 200, D-26180 Rastede, Phone: +49 4402 84248, Fax: +49 4402 939927, e-mail: r.telgmann@ cit-wulkow.de, URL: http://www.cit-wulkow.de

\section{KEYWORDS}

Virtual lab, in silico drug discovery, PBPK, systems biology, disease modeling

\section{SUMMARY}

Modeling and simulation in pharmacokinetics has has turned into the focus of pharmaceutical companies, driven by the emerging consensus that in silico predictions combined with in vitro data have the potential of significantly increasing insight into pharmacokinetic processes. To adequately support in silico methodology, software tools need to be user-friendly and, at the same time, flexible. In brief, the software has to allow the realization of modeling ideas that are beyond current knowledge - in the form of a virtual lab. We present and discuss the necessary design principles and concepts. These have been implemented in the software package MEDICI-PK to demonstrate its feasibility and advantages.

\section{PHARMACOKINETICS IN DRUG DISCOVERY}

The medical benefits of a drug depend not only on its biological effect at the target protein, but also on its "life cycle" within the organism - from its absorption into the blood, distribution to tissue and its eventual breakdown or excretion by the liver and kidneys. Pharmacokinetics is the study of the drug-organism interaction, in particular the investigation of absorption, distribution, metabolism, and excretion (ADME) processes [1,2]. Studying ADME profiles is widely used in drug discovery to understand the properties necessary to convert leads into good medicines [3,4].

As a result of studies in the late 1990s, indicating that poor pharmacokinetics (PK) and toxicity were important causes of costly late-stage failures in drug development, it has 
become widely appreciated that these areas should be considered as early as possible in the drug discovery process [3]. A great deal of in vitro data on physicochemical properties and specific ADME processes is already available at early stages of the drug discovery process. These data related to new drug candidates can be used in physiologically-based pharmacokinetic (PBPK) models to predict, analyze and optimize the pharmacokinetics of the compounds $[5,6]$. For instance, a combination of only five compound-specific parameters (fraction unbound in plasma, blood plasma ratio, intrinsic clearance, $\mathrm{pKa}$ and octanol water partition coefficient) and known species-related physiological parameters are required for the first PK estimates of an i.v. application [5]. Along the drug discovery process, more detailed compound-specific data and in vivo data are generated, upon which more detailed predictions and analyses can be made. The PBPK model approach is flexible, in the sense that it has the potential to be continuously updated in the light of new information, whether physiologic, disease, or drug related, including up- and down-regulation of critical components [11]. In particular, PBPK modeling has the advantage of being able to incorporate experimental animal data as well as in silico-derived and in vitro data into a coherent framework, from which meaningful and reliable assessments can be made [10].

It is worth noticing that in toxicology, physiologically-based models have a longer history and have been successfully applied, because of the almost exclusiver reliance upon animal data for the assessment of toxicological risk for humans [7-9].

\section{IN SILICO MODELING}

In the engineering sciences, computational approaches form an integral part in the developing process-some biological disciplines like, e.g. systems biology, have rather recently proceeded in the same direction [12,13]. Modeling and simulation help to analyze and understand large complex systems, in particular when the system comprises several subunits interacting in a strong and time-dependent manner. This is exactly the situation present in pharmacokinetics, where such subunits are typically physiological processes such as transport by the blood circulation, binding to macro-molecules, permeation through membranes, metabolism by the liver enzymes, excretion by the kidney and so on. While each of the processes is comparable simple, the overall behaviour is not.

There is no single established model underlying pharmacokinetics, and it will remain out of reach in the foreseeable future. This situation is completely different from, e.g., quantum mechanics or electrodynamics, where the Schrödinger equation or the Maxwell equations, respectively, are the basis and starting point for modeling and simulation attempts. Instead, the type of model needed will depend on the question to be resolved and, therefore, on the drug development stage at which the question is addressed. In silico approaches will continue to evolve rapidly, just as in vitro methods did during the last decade [10]. The quality and predictive power of new models will be judged in comparison with experimental data as well as in comparison with other models (as an example see, e.g., the study in [14]). A variety of different models for oral absorption are avaliable[15-19]. By comparing these absorption models for different test compounds, it would be possible to understand the "domain of applicability" of each model, i.e., under which conditions and for which class of compounds the models give the most reliable predictions. Partition coefficients are another example; there are different in silico methods to determine partition coefficients [20,21]. It would be interesting to compare, not only the different predicted partition coefficients, but also, in silico pharmacokinetic studies for a list of test compounds, based on these different partition coefficients. This would allow for a sensitivity analysis to identify the most critical partition 
coefficient. To support this type of comparative studies, flexibility in modeling will be crucial.

The present status of available software tools for modeling and simulation in pharmacokinetics has been thoroughly summarized in [11]. The software ranges from (i) generalpurpose high-level scientific software, such as Berkeley Madonna, Matlab, MLAB and Octave; (ii) bio-mathematical modeling software, like ADAPT II, ModelMaker, NONMEM, WinNonlin; (iii) toxicokinetic software, e.g., ACSL Toxicology Toolkit, SimuSolv and (iv) physiologically-based custom-designed software, such as GastroPlus, Pathway Prism, PKSim and Physiolab. As stated in [11], "it appears that there is an inverse relationship between user-friendliness and flexibility". However, combining flexibility with user-friendliness is exactly the domain of a virtual lab.

The modeling situation is comparable to the situation in systems biology $[12,13,22,23]$, where a large variety of modeling and simulation tools are available. Software tools like COPASI, E-CELL, Virtual Cell, and many more, have been designed to fulfill the needs of system biological modeling (a comprehensive list of software in this field can be found online under URL http://sbml.org). These tools are modular and open and allow for implementation of arbitrary models. Sometimes, typical reaction schemes are pre-implemented (like linear or Michaelis-Menten type kinetics), but flexibility is almost always retained. In principle, software tools developed in systems biology could be used in pharmacokinetics as well; however, they do not support the special structure/needs in pharmacokinetics. Jointly with the upcoming modeling efforts there has been a collective initiative of a number of research institutions to standardize models in order to facilitate exchange of models between different tools. As a result, the Systems Biology Markup Language (SBML) was created, a computerreadable format for representing models of biochemical reaction networks [24]. SBML is applicable to metabolic networks, cell-signaling pathways, regulatory networks and many others, and might also become interesting for PK.

In the light of our experience, we see the current status of modeling in pharmacokinetics comparable to the status of fields like, e.g., polymer chemistry at about 10-15 years ago. Since then, experts in these fields have experienced that even without definite $a$ priori knowledge of the "true" model and its parameters, the ongoing modeling process reveals such a tremendous insight into the system, that the modeling research usually pays off after a short time. At the same time, significant feedback between modeling progress and experimental methods could be observed. Sometimes new modeling ideas required better or newly-designed experiments; sometimes a breakthrough of analytical techniques enforced a more detailed modeling. We are convinced that this progress will take place in pharmacokinetics, in a similar way, in the near future. The development of the design principles and concepts of a virtual lab for PK has already shown that modeling principles from polymer chemistry, particle technology, catalysis and reaction engineering can be applied here in a structural way. After all, from a mathematical point of view, a pharmacokinetic model is not so different to a network of multiple-phase bio-reactors.

\section{THE VISION AND THE BENEFIT}

It is the emerging consensus that in silico predictions are no less predictive of what occurs in vivo than are in vitro tests, with the decisive advantage that much less investment in technology, resources and time is needed [10,25]. We believe that the combination of in vitro experiments and in silico modeling will dramatically increase the insight and knowledge 
about the relevant physiological and pharmacological processes in drug discovery. In the future, rather than performing separate target validation, biomarker identification, lead generation and optimization, candidate selection and preclinical development studies, these separate steps will be vertically integrated, accompanied by an in silico modeling process bringing together the knowledge gained from each of these steps into a disease-specific whole body model. The physiologically-based modeling approach offers a scientifically-defensible method (instead of just an educated guess) for the integration of these various pieces of information from in vitro studies and other preclinical information, in order to evaluate the outcome under various assumptions [26]. Each pharmaceutical company can build their own model tailored to their individual indications, knowledge and experience. Rather than modeling, analyzing and measuring plasma or tissue drug concentrations, these diseasespecific whole body models allow the study of effect-specific processes. The modeling process will benefit greatly from the systems biology community and their experience, insight and available models on metabolic pathways, gene-regulatory networks and signaling pathways [12,22-24,27]. As stated in [13], the most feasible application of systems biology research is to create a detailed model of cell regulation, focused on particular signaltransduction cascades and molecules to provide system-level insights into mechanism-based drug discovery (e.g., [28]). Such models may help to identify feedback mechanisms that offset the effects of drugs and predict systemic side effects. Many of the necessary foundations have nearly been laid; however, there is no flexible, application specific and user-friendly software tool available that really supports this process in an open way.

\section{THE VIRTUAL LAB APROACH}

In mathematical terms, a PBPK model consists of a set of differential equations describing the ADME processes of one or more compounds in a system of more than a dozen organs, each being possibly further subdivided into a number of phases (e.g., erythrocytes, plasma, interstitial and cellular space). Coding such a system in a programming language is possible, however cumbersome and error prone, and a subsequent extension or change of the model will even be more complicated. For the design of a virtual lab, it is obvious that on the one hand, the software tools should allow for the input of models in a very open way, yet on the other hand, should not force the user to enter the whole set of differential equations. Thus, an implementation of a static model is prohibitive, since it contradicts the requirement on openness of the software tool; furthermore, a purely equation-based approach is in contrast with the requirement of user-friendliness. However, unfortunately these are two typical approaches often met in engineering software packages. Instead, the requirements on openness and user-friendliness have to be fulfilled by the use of a sophisticated modular structure implemented by means of modern software concepts (cf. e.g [29]).

The virtual lab should allow the implementation of a hierarchy of models suitable for the integration of as much knowledge (data) as available into the model, i.e., few in vitro data at the beginning, followed by more detailed in vitro data and in vivo data later on. At any point, the model should be completely transparent; there should be neither hidden calculations nor parameters. From our experience, this is an important point since transparency is a prerequisite for confidence in the modeling process. In addition, as stated in [10], "Software producers need to improve transparency and state clearly the assumptions underlying their predictive approach". Moreover, the integration of system biological data (e.g. from the target finding and validation process) should be possible, proceeding towards the simulation of biomarkers and effect-related processes. Of course, the use of state-of-the-art numerical algorithms and the possibility to easily and flexible analyze the simulation output and the 
comparison of different models on the basis of their simulation results is self-evident. This is important for the a posteriori evaluation of early/coarse models in comparison with late/refined models, as well as for the design of new models, e.g., for oral absorption. A modular structure should facilitate modeling of drug-drug-interactions and support the development of disease specific models for the design of virtual "patients" (humans as well as animals) that can be exploited in the drug discovery process.
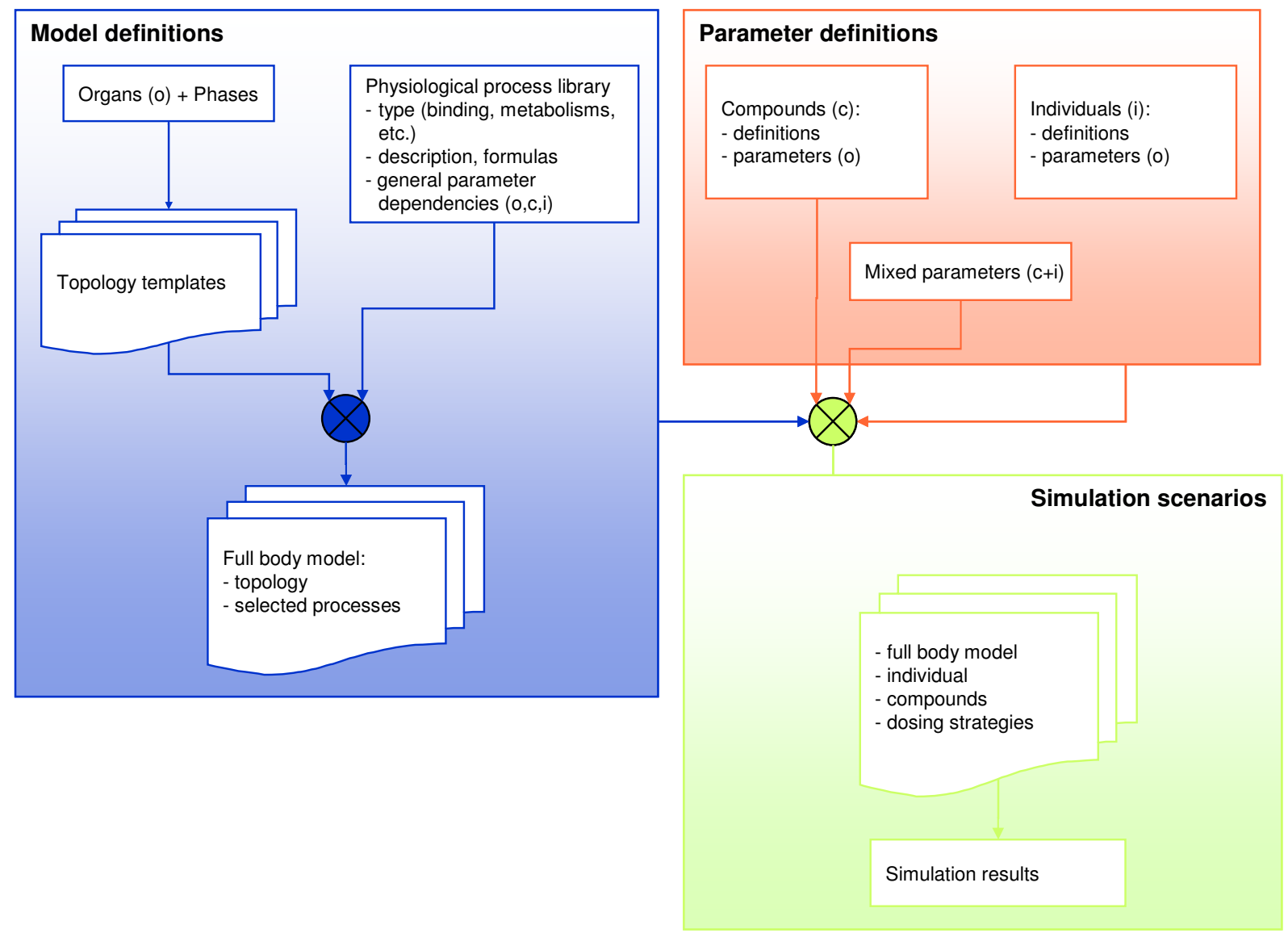

Figure 1: Modular structure of MEDICI-PK: All definitions, models and parameters can be entered and changed by the user and are not limited. Based on the defined framework (topology, model library, list of substances and individuals) the components of a model form a so-called "Simulation object" describing one simulation scenario. A project may consist of an open list of all shown structures; simulation results from totally different models can easily be compared.

\section{MEDICI-PK}

The above derived design principles and concepts have been realized for the first time in the virtual lab MEDICI-PK (see Table 1), demonstrating its feasibility and illustrating the advantages and benefits of the concept. The underlying structure can be seen in Fig. 1. A crucial point is the so-called orthogonality of the model and the parameters. The compounds and the individuals (representing either a typical, healthy species or a disease type) are basically a collection of parameters and the source of input for the models. The models, however, are established independently from the compound and the individual. They are based on typical physiological processes, like protein binding, diffusion across the membrane, metabolism and so on. Each of these typical processes can be represented by a set of (differential) equations, without setting up the whole body model. This guarantees that the 
underlying physiological processes are accessible, extendable and fully transparent. When performing a case study, one simply has to choose the underlying whole body physiologically-based model, the individual (and therefore the species) and the compound (Fig. 2). This way, greatest flexibility is retained. For instance, simulating the same compound with the same model for a different species requires just two "clicks" (assuming, of course, that the necessary model parameters are available). To give another example: assume we want to simulate a pro-drug, its metabolite (the drug) and a potential interaction of the drug with another compound in the kidney (thinking of oseltamivir, oseltamivir phosphate (Tamiflu) and probenecid [30,31]). Then, in a first step one needs to choose a model, an individual and one of the compounds at a time to simulate their pharmacokinetics. Now, in a second step, the interaction needs to be included into the model. This is realized by defining two new basic physiological models: (i) metabolism in the liver, taking explicitly the production of the metabolite into account, (ii) competitive excretion in the kidney (to build this model, one can use the insight from competitive metabolism). Choosing these two basic physiological models in the whole body physiologically-based pharmacokinetic model, the overall interaction of the three compounds can now be studied by simulation. This nicely illustrates who easily interactions of different compound can be integrated into the model.

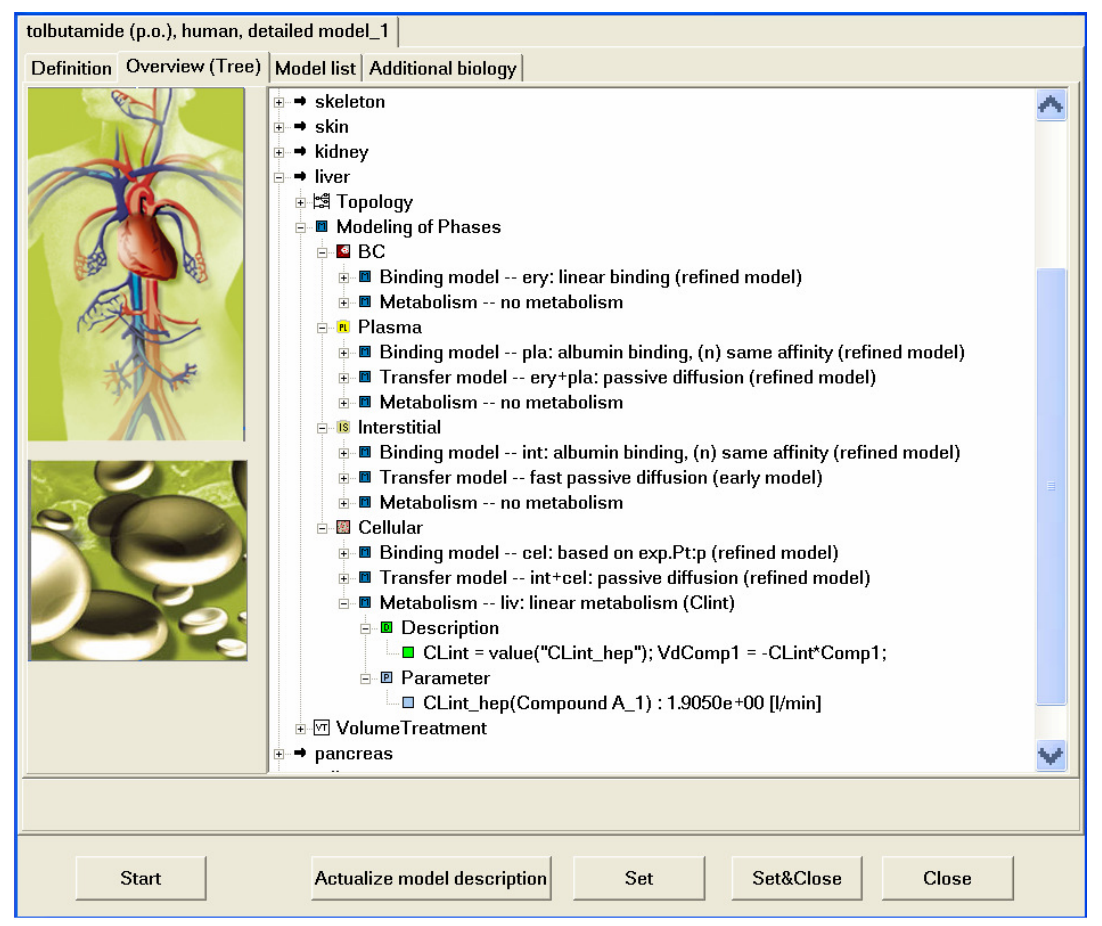

Figure 2: A Medici-PK simulation object summarizing all settings and parameters of a single simulation scenario. All details are assessable and can be changed in the model tree.

A number of different models and simulation studies have already been realized in MEDICIPK in this way, e.g. a model on Cyclosporine A [32], a detailed model on the PK of tolbutamide and a generic PBPK model for early drug development (following [5,33]). Immediately extensions of the existing models have been studied (Fig. 3), e.g. incorporation of metabolites, comparison of different binding and tissue distribution models, drug-druginteraction studies, comparison between different species (human, rat, and so on). 


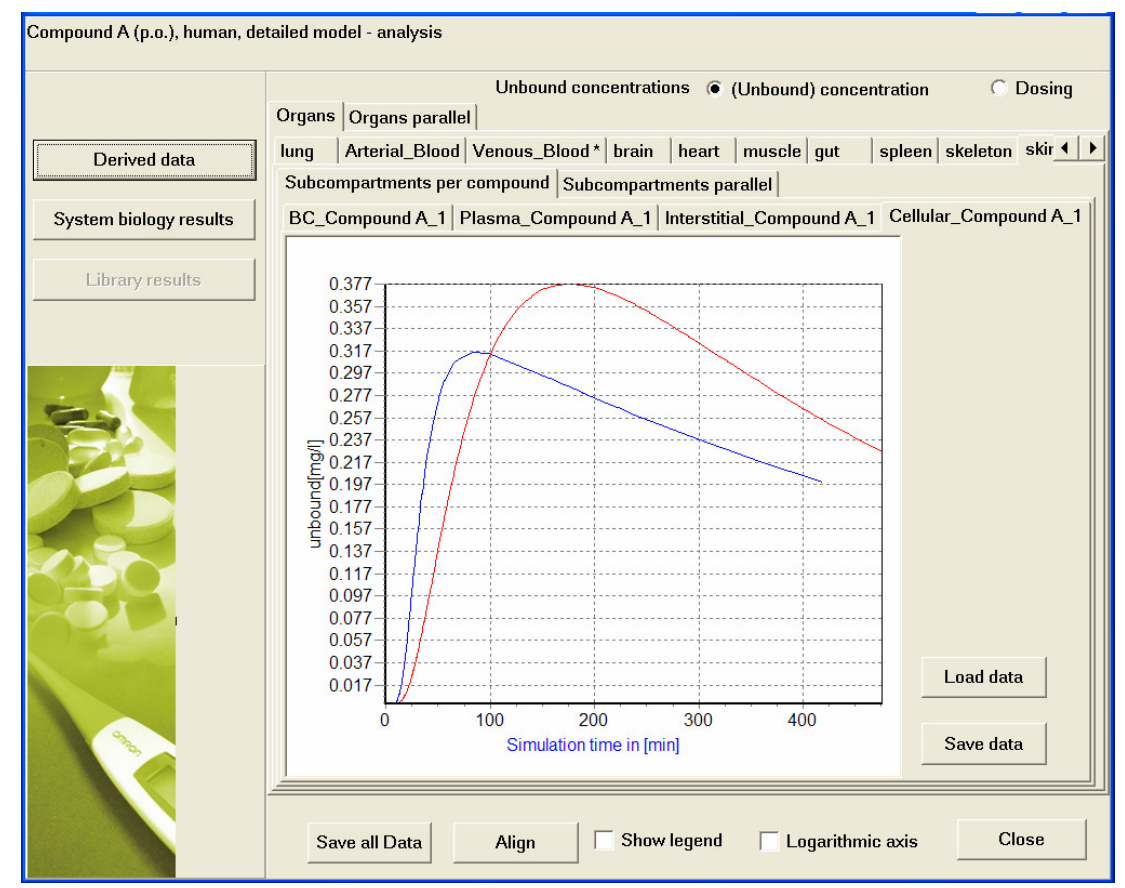

Figure 3: Typical simulation output of a MEDICI-PK case study. The results for all organs and phases can be visualized and compared. The graphic shows concentration time curves, where the blue line correspond to the results obtained by use of a more refined model in comparison to the result of a coarser model (red line).

\section{CONCLUSION}

This article presents the design principles and concepts of a virtual lab approach implemented in the program package Medici-PK - that supports in silico modeling and simulation in PK/PD. The concept is especially tailored to serve the needs in drug discovery. Based on our experience in other fields of chemistry and biology we see a high potential for in silico $\mathrm{PK} / \mathrm{PD}$ approaches in the drug discovery and development process if properly realized. When starting the modeling and simulation activities, there will soon be a first benefit: modeling eventually brings together people with different backgrounds and different company internal affiliations-thereby increasing the flux of information across different process stages (in this case ideally between the research, development and clinical phase). Moreover, in the process of developing an appropriate model, important modeling questions have to be addressed. What are the most relevant physiological processes and mechanisms? Is there any detailed knowledge about these processes or are they (partially) unknown? Are there competing hypotheses about important mechanisms? A further benefit is the integration of in silico, in vitro or in vivo data into a coherent framework by which they both (model and data) undergo a kind of consistency check. Once a first model has been established, hypotheses can be generated and tested by simulation. Having gained first confidence in the model, predictions generated by simulation studies will influence the design of future experimental studies (in vitro and in vivo). As can be seen in many different fields this feedback loop is often the beginning of a substantial change in the quality of models and understanding of the processes of interest.

It is important to note that building a comprehensive and predictive model usually cannot be realized in a few days. The decision to do modeling at best implies a long-term directive. It is a process gaining and accumulating new insights from the very beginning, but the "final" model will hardly look like the first attempts or approaches published elsewhere. For PK- 
modeling this means that a compound should be continuously monitored in silico along its way through the research, development and clinical phases. Once established, a good model can be 're-used' for every new drug candidate thereby decreasing the amount of work to be invested per compound and ensuring an information flux back from the (data rich) clinical phase to the target finding/validation and lead generation phase.

Table 1: Important feature of the virtual lab MEDICI-PK (for more details, see text)

\begin{tabular}{|c|c|c|}
\hline Property & Description & Realization in MEDICI-PK \\
\hline User-friendliness & $\begin{array}{l}\text { Assistance of the specific needs in } \\
\text { physiological-based PK modeling }\end{array}$ & $\begin{array}{l}\text { Support of model building, hypothesis testing, } \\
\text { user defined output variables, data import from } \\
\text { SBML, data export to Excel }\end{array}$ \\
\hline Application-specificity & $\begin{array}{l}\text { Software in terms of a } \\
\text { pharmacokinetic-specific } \\
\text { language }\end{array}$ & $\begin{array}{l}\text { Application motivated design of underlying } \\
\text { software structure; compounds, species, organs, } \\
\text { dosing schemes, simulation objects as typical } \\
\text { building blocks }\end{array}$ \\
\hline Modularity & $\begin{array}{l}\text { Characterization of the overall PK } \\
\text { model in terms of simple, } \\
\text { independent modules that can be } \\
\text { used in multiple contexts }\end{array}$ & $\begin{array}{l}\text { Extendable list of modules (physiological } \\
\text { processes like binding, transfer, metabolism, } \\
\text { metabolic pathways etc) in a model basis, } \\
\text { definition of each full body PK model in terms } \\
\text { of the underlying physiological processes. }\end{array}$ \\
\hline $\begin{array}{l}\text { Orthogonal design and } \\
\text { flexibility }\end{array}$ & $\begin{array}{l}\text { Separation of model constituents } \\
\text { that are independent of each other }\end{array}$ & $\begin{array}{l}\text { Independent definition of compound-specific } \\
\text { data, species-specific physiological parameters } \\
\text { and mathematical models to archive largest } \\
\text { flexibility }\end{array}$ \\
\hline Openness & $\begin{array}{l}\text { No limitation for adding new types } \\
\text { of parameters, compounds, organs, } \\
\text { species, physiological models etc. }\end{array}$ & $\begin{array}{l}\text { Easily extendable lists of parameters, } \\
\text { compounds, organs, species, physiological } \\
\text { models, dosing schemes etc. }\end{array}$ \\
\hline Transparency & $\begin{array}{l}\text { No hidden calculations or } \\
\text { parameters }\end{array}$ & $\begin{array}{l}\text { All parameters and models are directly } \\
\text { assessable and editable }\end{array}$ \\
\hline $\begin{array}{l}\text { Computational } \\
\text { efficiency }\end{array}$ & $\begin{array}{l}\text { Use of fast and accurate numerical } \\
\text { techniques }\end{array}$ & $\begin{array}{l}\text { Integration of state-of-the-art numerical } \\
\text { algorithms }\end{array}$ \\
\hline
\end{tabular}

\section{ACKNOWLEDGEMENT}

This research has been supported by the DFG Research Center MATHEON "Mathematics for key technologies: Modelling, simulation, and optimization of real-world processes", Berlin.

\section{REFERENCES}

[1] Kwon, Y. (2001) Handbook of Essential Pharmacokinetics, Pharmacodynamics and Drug Metabolism for Industrial Scientists, Kluwer Academic/Plemun Publishers

[2] Schoenwald, R.D., ed. (2002) Pharmacokinetics in Drug Discovery and Development. CRC Press

[3] Waterbeemd, H. van, Gifford, E. (2003) ADMET in silico modelling: towards prediction paradise? Nature 2, 192-204 
[4] Selick, H.E. et al. (2002) The emerging importance of predictive ADME simulation in drug discovery. DDT 7, 109-116

[5] Poulin, P. and Theil, F.-P. (2002) Prediction of Pharmacokinetics prior to In Vivo Studies. II. Generic Physiologically Based Pharmacokinetic Models of Drug Disposition. J. Pharm. Sci. 91, 1358-1370

[6] Lüpfert, C., and Reichel, A. (2006) Development and Application of Physiologically Based Pharmacokinetic-Modeling Tools to Support Drug Discovery. Chem. Biodiversity 2, $1462-1486$

[7] Reddy, M.B. et al. , eds. (2005) Physiologically Based Pharmacokinetic Modeling. Wiley

[8] Anderson, M.E. (1995) Development of physiologically based pharmacokinetic and physiologically based pharmacodynamic models for applications in toxicology and risk assessment. Tox. Letters, 79, 35-44

[9] Gerlowski, L.E., and Jain, R.K. (1983) Physiologically based pharmacokinetic modeling: Principles and applications. J. Pharm. Sci., 72, 1103-1127

[10] Boobis, A. et al. (2002) In silico prediction of ADME and pharmacokinetics. Report of an expert meeting organised by COST B15. Eur. J. Pharm. Sci. 17, pp. 183-193

[11] Rowland, M. et al. (2004) Physiologically Based Pharmacokinetics in Drug Development and Regulatory Science: A Workshop Report (Georgetown University, Washington, DC, May 29-30, 2002), AAPS PharmSci, 6,. 1-12.

[12] Kitano, H, ed. (2001) Foundations of Systems Biology, MIT Press

[13] Kitano, H (2002) Systems Biology: A Brief Overview. Science, 295, pp.1662-1664

[14] Luttringer, O. et al. (2003) Physiologically Based Pharmacokinetic (PBPK) Modeling of Disposition of Epiroprim in Humans. J. Pharm. Sci. 92, 1990-2007

[15] Yu, L.X. and Amidon, G.L. (1999) A compartmental absorption and transit model for estimating oral drug absorption. Int. J. Pharm. 186, 119-125

[16] Agoram, B. et al (2001) Predicting the impact of physiological and biochemical processes on oral drug bioavailability. Adv. Drug. Del. 50., S41-S67

[17] Kimura, T. and Higaki, K. (2002) Gastrointestinal transit and drug absorption. Biol. Pharm. Bull 25, 149-164

[18] Weiß, L. (1996) A novel extravascular input function for the assessment of drug absorption in bioavailability studies. Pharm. Res. 13, 1547-1553.

[19] Willmann, S. et al. (2003) A physiological model for the estimation of the fraction dose absorbed in humans. J. Med. Chem. 47,. 4022-4031 
[20] Poulin, P, Theil, F.-P (2000) A priori prediction of tissue:plasma partition coefficients of drugs to facilitate the use of physiologically-based pharmacokinetic models in drug discovery. J. Pharm. Sci. 89, 16-34

[21] Rodgers, T., et al. (2005) Physiologically based pharmacokinetic modeling I: Predicting the tissue distribution of moderate-to-strong bases. J. Pharm. Sci., 94, 1259-1276

[22] Fall, C.P. et al. (2002) Computational Cell Biology, Springer, New York

[23] Keener, J.P. and Sneyd, J. (1998) Mathematical Physiology, Interdisciplinary Applied Mathematics, Vol. 8, Marsden J.E, Sirovich, L, and Wiggens, S. (eds.), Springer, New York

[24] Hucka, M. et al. (2003) The systems biology markup language (SBML): a medium for representation and exchange of biochemical network models. Bioinformatics 19, 524-531

[25] Modi, S. (2004) Positioning ADMET in silico tools in drug discovery. DDT, 9, pp. 14-15

[26] Olivier, R.E., Jones, A.F., Rowland, M. (2001) J. Pharmacokinet. Biopharm. 28, 27-55

[27] Bugrim, A. et al. (2004) Early prediction of drug metabolism and toxicity: systems biology approach and modeling. DDT, 9, 127-135

[28] Gibbs, J. B. (2000) Mechanism-Based Target Identification and Drug Discovery in Cancer Research. Science 287, 1969-1973

[29] Busch, M., Müller, M., Wulkow, M (2003). The Use of Simulation Techniques in Developing Kinetic Models for Polymerization. Chem. Eng. Technol, 26, 1031-1040

[30] Hill, G. (2002) The anti-influenza drug oseltamivir exhibits low potential to induce pharmacokinetics drug interactions via renal secretion --- correlation fo in vivo and in vitro studies. Drug Metab. Dispos., 30, 13-19

[31] Butler, D (2005) Wartime tactic doubles power of scarce bird-flu drug. Nature, 438, 7064-7066

[32] Kawai, R. et al. (1998) Physiologically based pharmacokinetics of cyclosporine A:

Extension to tissue distribution kinetics in rats and scale-up to human. J Pharmacol Exp Ther 287, 457-468

[33] Theil, F-P. et al. (2003) Utility of physiologically based pharmacokinetic models to drug development and rational drug discovery candidate selection. Tox. Letters 138, 29-49 